\title{
Sedation With Midazolam After Cardiac Surgery in Children With and Without Down Syndrome: A Pharmacokinetic-Pharmacodynamic Study
}

OBJECTIVES: To compare the pharmacokinetics and pharmacodynamics of IV midazolam after cardiac surgery between children with and without Down syndrome.

DESIGN: Prospective, single-center observational trial.

SETTING: PICU in a university-affiliated pediatric teaching hospital.

PATIENTS: Twenty-one children with Down syndrome and 17 without, 3-36 months, scheduled for cardiac surgery with cardiopulmonary bypass.

INTERVENTIONS: Postoperatively, nurses regularly assessed the children's pain and discomfort with the validated COMFORT-Behavioral scale and Numeric Rating Scale for pain. A loading dose of morphine $(100 \mu \mathrm{g} /$ $\mathrm{kg}$ ) was administered after coming off bypass; thereafter, morphine infusion was commenced at $40 \mu \mathrm{g} / \mathrm{kg} / \mathrm{hr}$. Midazolam was started if COMFORTBehavioral scale score of greater than 16 and Numeric Rating Scale score of less than 4 (suggestive of undersedation). Plasma midazolam and metabolite concentrations were measured for population pharmacokineticand pharmacodynamic analysis using nonlinear mixed effects modeling (NONMEM) (Version Vl; GloboMax LLC, Hanover, MD) software.

MEASUREMENTS AND MAIN RESULTS: Twenty-six children (72\%) required midazolam postoperatively (15 with Down syndrome and 11 without; $p=1.00)$. Neither the cumulative midazolam dose $(p=0.61)$ nor the time elapsed before additional sedation was initiated $(p=0.71)$, statistically significantly differed between children with and without Down syndrome. Population pharmacokinetic and pharmacodynamics analysis revealed no statistically significant differences between the children with and without Down syndrome. Bodyweight was a significant covariate for the clearance of $1-\mathrm{OH}$-midazolam to $1-\mathrm{OH}$-glucuronide $(p=0.003)$. Pharmacodynamic analysis revealed a marginal effect of the midazolam concentration on the COMFORT-Behavioral score.

CONCLUSIONS: The majority of children with and without Down syndrome required additional sedation after cardiac surgery. This pharmacokinetic and pharmacodynamic analysis does not provide evidence for different dosing of midazolam in children with Down syndrome after cardiac surgery.

KEY WORDS: cardiac surgical procedures; down syndrome; intensive care; midazolam; pharmacokinetics; sedation

n 1887, John Langdon Down (1) was the first to describe the altered reaction to surgical procedures in individuals with Down syndrome. Later, chart review studies showed that intraoperative dosing of opioids did not
Abraham J. Valkenburg, MD, PhD ${ }^{1,2}$

Sebastiaan C. Goulooze, $\mathrm{MSc}^{3}$

Cormac V. Breatnach, MD ${ }^{4}$

Ron A. A. Mathôt, PharmD, PhD ${ }^{5}$

Dick Tibboel, MD, PhD²

Monique van $\mathrm{Dijk}, \mathrm{PhD}^{2,6}$

Catherijne A. J. Knibbe, PharmD, $\mathrm{PhD}^{3,7}$

Mariska Y. M. Peeters, PharmD, $\mathrm{PhD}^{7}$

Copyright ( $\odot 2020$ by the Society of Critical Care Medicine and the World Federation of Pediatric Intensive and Critical Care Societies

DOI: $10.1097 /$ PCC. 0000000000002580 
differ between children with and without Down syndrome (2) although those with Down syndrome more often received sedatives and muscle relaxants after cardiac surgery than children without Down syndrome (3). It has consistently been shown that the pharmacokinetics and pharmacodynamics of morphine are not different between children with and without Down syndrome after cardiac surgery $(4,5)$.

In the Republic of Ireland, the prevalence of Down syndrome is 1:546 live births (6). Approximately $54 \%$ of infants with Down syndrome have a congenital heart defect, often requiring cardiac surgery at a young age (7). To ensure their optimal postoperative treatment, it is highly relevant to study the pharmacokinetics and pharmacodynamics of the sedative agents employed. Midazolam is still the most used sedative agent in pediatric intensive care after cardiac surgery, despite wide variability in pharmacokinetics and the risk for delirium (8).

The aim of this study was to prospectively compare the pharmacokinetics of midazolam between children with and without Down syndrome after cardiac surgery and to link the midazolam concentrations to the pharmacodynamics by means of quantifiable distress assessments.

\section{MATERIALS AND METHODS}

\section{Subjects and Setting}

We performed an observational, prospective comparative cohort study at the Department of Anesthesia and Intensive Care Medicine of Our Lady's Children's Hospital, Dublin. The study protocol had been approved by the local medical ethics review board. Written informed consent for participation of their child in the study was obtained from the parents preoperatively. The analysis of the pharmacodynamics and pharmacokinetics of IV morphine in this cohort has been published previously (4).

Children between 3 and 36 months old, admitted to the ICU after cardiac surgery with cardiopulmonary bypass for atrial septal defect, ventricular septal defect, atrioventricular septal defect, or Tetralogy of Fallot repair, were eligible for participation in the study. The exclusion criteria were as follows: epilepsy, cerebral palsy, birth asphyxia, history of cardiothoracic surgery through sternotomy, preoperative mechanical ventilation, preoperative treatment with morphine or midazolam, and extracorporeal membrane oxygenation treatment after cardiopulmonary bypass.

Data were collected from the moment of the child's arrival in the operating theatre until one of the following events: a switch from IV to oral morphine, discharge to the ward, a procedure requiring general anesthesia, and reintubation for any reason other than oversedation.

\section{General Anesthesia}

Children received a standardized general anesthesic regimen without premedication as previously described (4). After discontinuation of cardiopulmonary bypass, a morphine loading dose $(100 \mu \mathrm{g} / \mathrm{kg})$ was administered, and a morphine infusion was commenced at $40 \mu \mathrm{g} / \mathrm{kg} / \mathrm{hr}$.

\section{Postoperative Intensive Care Management}

All patients received standardized postoperative pain and distress management titrated to pain. The first 24 hours after the surgery, the child's pain and distress were assessed every 2 hours using the COMFORTBehavioral scale (COMFORT-B scale) and Numeric Rating Scale (NRS). Thereafter, scores were obtained every 4 hours and on indication (e.g., monitoring the effect of an intervention). Further information on the validity of these pain and distress assessment tools can be found in the original articles describing children with and without Down syndrome (9-13).

At arrival at the ICU, the morphine infusion was continued at $40 \mu \mathrm{g} / \mathrm{kg} / \mathrm{hr}$. Additional morphine was given (20-40 $\mu \mathrm{g} / \mathrm{kg}$ bolus dose) if the COMFORT-B score was greater than or equal to 17 in combination with a NRS score greater than or equal to 4 (9)-indicating moderate to severe pain. Undersedation was defined as COMFORT-B score greater than 16 in combination with a NRS score less than 4 and considered as an indication to start midazolam. Midazolam boluses $(50-100 \mu \mathrm{g} / \mathrm{kg}$ ) were prescribed as needed with escalation to a midazolam infusion $(1-2.5 \mu \mathrm{g} / \mathrm{kg} / \mathrm{min})$ if still further sedation was needed within 1 hour after the bolus administration.

All patients received three doses of IV acetaminophen for the first 24 hours $(7.5 \mathrm{mg} / \mathrm{kg}$ for children $<10 \mathrm{~kg}$ and $15 \mathrm{mg} / \mathrm{kg}$ for children $>10 \mathrm{~kg}$ ). At the discretion of the attending physician, rescue analgesics could be administered: either clonidine IV $(1 \mu \mathrm{g} / \mathrm{kg})$ or ibuprofen $(10 \mathrm{mg} / \mathrm{kg})$ as a suppository. 


\section{Samples for Pharmacokinetic Analysis}

Arterial blood sampling was scheduled as follows: just before the first midazolam dose $(t=0)$ and next at $t=30$ 60 minutes, $\mathrm{t}=4-8$ hours, and $\mathrm{t}=24$ hours. Additional samples were obtained daily at $8.00 \mathrm{Am}$ and once just before the end of the study. Blood samples $(1.0 \mathrm{~mL})$ were centrifuged, and plasma was stored at $-80^{\circ} \mathrm{C}$. Details on the analysis of the midazolam, 1-OH-midazolam, 4-OH-midazolam, 1-OH-midazolam-glucuronide levels using liquid chromatography with tandem mass spectroscopy can be found in the supplementary material (Supplemental Digital Content 1, http://links. lww.com/PCC/B533).

\section{Population Pharmacokinetic Analysis}

The population pharmacokinetic analysis was performed using nonlinear mixed effect modelling (NONMEM, Version VI; GloboMax LLC, Hanover, $\mathrm{MD})$ by use of the first-order conditional estimation with $\eta-\varepsilon$ interaction and ADVAN6 TOL5 (part of NONMEM software). The data were visualized using S-plus (Version 6.2; Insightful software, Seattle, WA). Model building was consisted of these four different steps: 1) selection of the structural model (one-, two-, or three-compartment model), 2) choice of the statistical model, 3) covariate analysis, and 4) model evaluation. Discrimination between different models was made by comparison of the objective function. A value of $p$ less than 0.01 , representing a decrease of 6.63 points in the objective function, was considered statistically significant. For more details, we refer to the supplementary material (Supplemental Digital Content 1, http://links.lww.com/PCC/B533).

\section{Covariate Analysis}

To visualize potential relationships, the covariates bodyweight, age, sex, Down syndrome, aspartate transaminase (AST), alanine transaminase (ALT), bilirubin, $\mathrm{C}$-reactive protein (CRP), and creatinine were plotted subsequently against the individual post hoc variable estimates and the weighted residuals. On the basis of these plots, covariates were tested for their influence. Starting from the basic model without covariates, the covariate model was first built up using forward inclusion $(p<0.005$ representing a decrease of 7.88 points in objective function). Finally, after forward inclusion, a backward exclusion procedure was applied to justify the inclusion of a covariate $(p<0.005)$.

\section{Model Validation}

The internal validity of the population pharmacokinetic model was assessed by the bootstrap resampling method, that is, repeated random sampling to produce another dataset of the same size but with a different combination of individuals. Variables obtained with the bootstrap replicates (500 times) were compared to the estimates obtained from the original dataset.

\section{Simulations}

To compare the pharmacokinetic results from the current study with those of other pharmacokinetic models on midazolam in children, simulations were performed using the final modal and these two population pharmacokinetic models from the literature. One of these other studies reported midazolam pharmacokinetics in critically ill children (0-18 yr old; median age 5.1 mo) (14); the other reported this for nonventilated otherwise healthy children after craniofacial surgery (3-24 mo old) (15).

\section{Pharmacodynamic Analysis}

Input for the pharmacodynamic model consisted of the COMFORT-B scores at start of the midazolam infusion, routine scores, and scores obtained directly after an intervention. The COMFORT-B scores were modelled as ordered categorical data with three levels: undersedation (COMFORT-B score > 16), adequate sedation (COMFORT-B score between 11 and 16), and oversedation (COMFORT-B score below 11). Model estimation was performed using the Laplace estimation method in NONMEM. Details on this population pharmacodynamic analysis are presented in the supplementary material (Supplemental Digital Content 1, http://links.lww.com/PCC/B533).

\section{Statistical Analysis}

Univariate data were analyzed using SPSS version 23.0 (IBM, Chicago, IL). Nominal data were compared using the chi-square test (or Fisher exact test in the case of low predicted cell counts). Continuous data are presented as median (interquartile range [IQR]), and data for the children with and without Down 
syndrome were compared with the Mann-Whitney $U$ test. All $p$ values are two sided, and a value of less than 0.05 is considered statistically significant.

\section{RESULTS}

Thirty-eight children participated (Supplementary Fig. 1, Supplemental Digital Content 1, http://links. lww.com/PCC/B533) that is, 21 with Down syndrome and 17 without (Table 1). Parents of one child with Down syndrome did not give consent for the pharmacokinetic blood samples. Data of two children without Down syndrome were excluded from the analysis (one could not be weaned off cardiopulmonary bypass and was commenced on extracorporeal membrane oxygenation, the other because of inadvertent disconnection of the IV catheter for an unknown period during transport from theatre to the PICU).

Twenty-six subjects (72\%), 15 with Down syndrome and 11 without, received midazolam postoperatively. The median (IQR) time from arrival in the PICU to administration of the first dose was 6 hours (5-14hr) for the children without Down syndrome and 10 hours (5-15 hr) for children with Down syndrome $(p=0.71)$. The median number of midazolam boluses per patient was 5 (IQR 0-7) for the children without Down syndrome and 2 (IQR 0-9) for the children with Down syndrome $(p=0.68)$. A midazolam infusion was administered to seven children (33\%) with Down syndrome and seven children (47\%) without Down syndrome, $p$ equals to 0.42 . The median cumulative dose of midazolam per patient was 1,037 (IQR 101-2,516) microgram per kilogram during a median intensive care admission of 4 days (IQR 3-7 d). The cumulative midazolam dose did not statistically significantly differ between children with and without Down syndrome $(p=0.61)$. In two subjects without Down syndrome, midazolam infusion was stopped shortly after extubation. In one subject with Down syndrome and one without, a midazolam bolus was given after extubation.

The children who needed midazolam postoperatively had longer cardiopulmonary bypass times during surgery compared with those who did not require midazolam (median $119 \mathrm{~min}$ [IQR 100-136 min] vs $80 \mathrm{~min}$ [IQR 66-120 min]; $p=0.04$ ). The children who received midazolam stayed longer in the PICU—with a median (IQR) duration of 4 days (3-7 d) days versus 2 days (1-3 d) for those who did not receive midazolam $(p=0.01)$.

\section{Pharmacokinetics of Midazolam}

Serumconcentrationsofmidazolam,1-OH-midazolam, 4-OH-midazolam, and 1-OH-midazolam-glucuronide exceeded lower limit of quantification (LLQ) in 147, $141,81,156$ samples and were below LLQ in respectively $10.4 \%, 14 \%, 50.9 \%$, and $6.0 \%$ of the samples. The pharmacokinetic model used is schematically depicted in Supplementary Figure 2 (Supplemental Digital Content 1, http://links.lww.com/PCC/B533). Log transformed midazolam data were best described with a two-compartment model, parameterized in terms of the volume of the central compartment (V1), intercompartmental clearance between central and peripheral volume (Q), peripheral volume (V2), clearance to 1-OH-midazolam (CL1), and clearance to 4-OH-midazolam (CL4). The metabolites 1-OH-midazolam and 4-OH-midazolam were best described with a one-compartment model, whereas 1-OH-midazolam-glucuronide was best described with a two-compartment model. The residual errors were described with a proportional error model.

Table 2 lists the pharmacokinetic variable estimates and the results from the bootstrap analysis of the final model. The goodness of fit plots of the final model indicated a successful characterization of the data (SupplementaryFig.3, SupplementalDigitalContent 1, http://links.lww.com/PCC/B533). Bodyweight was a significant covariate for the clearance of 1-OH-midazolam to 1-OH-glucuronide (CL2)implemented as a linear function (decrease in objective function of 8.77 points, $p=0.003$ ). Supplementary Figure 4 (Supplemental Digital Content 1, http://links. lww.com/PCC/B533) shows the relationship between bodyweight and CL2. Bodyweight as a covariate for peripheral volume of midazolam was rejected because the volumes of distribution of 1-OH-glucuronide could not be estimated anymore with adequate precision. Age was a significant covariate for the central volume of midazolam (decrease in objective function $[\Delta \mathrm{OF}]=9.71$, $p=0.002$ ), but incorporation of bodyweight on CL2 and age on V1 gave unstable results for the bootstrap analysis. Down syndrome was not a significant covariate on any of the pharmacokinetic variables. Furthermore, sex as well as markers of inflammation 
TABLE 1.

Patient Characteristics

\begin{tabular}{|c|c|c|c|}
\hline Patient Characteristics & $\begin{array}{l}\text { Down Syndrome } \\
(n=21)\end{array}$ & $\begin{array}{l}\text { Controls } \\
(n=15)\end{array}$ & $\mathbf{p}$ \\
\hline Male sex, $n(\%)$ & 7 (33) & $8(53)$ & 0.23 \\
\hline Postnatal age, d, median (IQR) & $175(127-272)$ & $204(123-235)$ & 0.50 \\
\hline Procedure, $n$ & & & $<0.001^{a}$ \\
\hline Atrial septal defect & 1 & 0 & \\
\hline Ventricular septal defect & 4 & 5 & \\
\hline Atrioventricular septal defect & 15 & 1 & \\
\hline Tetralogy of Fallot & 0 & 9 & \\
\hline Atrioventricular septal defect and tetralogy of fallot & 1 & 0 & \\
\hline Cardiopulmonary bypass time, min, median (IQR) & $115(80-127)$ & $111(72-134)$ & $0.92^{b}$ \\
\hline $\begin{array}{l}\text { Risk Adjustment for Surgery for Congenital Heart } \\
\text { Disease-1 score 3, } n(\%)\end{array}$ & $16(76)$ & $1(7)$ & $<0.001^{a}$ \\
\hline Morphine cumulative dose on day 1 in $\mu \mathrm{g}$, median (IQR) & $936(705-1,076)$ & $956(845-1,074)$ & 0.55 \\
\hline Pediatric Logistic Organ Dysfunction score day 1, median (IQR) & $1.15(0.1-1.3)$ & $1.3(0.1-1.3)$ & 0.78 \\
\hline Duration of postoperative mechanical ventilation, hr, median (IQR) & $26(19-29)$ & $26(17-50)$ & 0.82 \\
\hline Duration of intensive care admission, $d$, median (IQR) & $4(2-7)$ & $4(2-6)$ & 0.92 \\
\hline
\end{tabular}

IQR $=$ interquartile range

${ }^{a} p$ values are two-sided. A value of $<0.05$ is considered statistically significant.

(CRP), liver tests (AST, ALT, bilirubin), and kidney function (creatinine) could not be identified as significant covariates.

The midazolam concentrations established in the current study compared better with those reported by Vet et al (14) than those reported by Peeters et al (15) (Fig. 1).

\section{Pharmacodynamics}

A total of 609 COMFORT-B and NRS scores were collected. The median COMFORT-B score was 13 (IQR 12-16) for the children with Down syndrome and 14 (IQR 12-24) for the children without Down syndrome $(p=0.78)$. The median NRS was 2 (IQR $0-2)$ for the children with Down syndrome and 2 (IQR 0-2) for the children without $(p=0.57)$.

Immediately before the first midazolam administration, the median COMFORT-B score was 18 (IQR 17-19). There were no differences in individually predicted midazolam levels and COMFORT-B scores between the children with and without Down syndrome (Supplementary Fig. 5, Supplemental Digital Content 1, http://links.lww.com/PCC/B533).

To demonstrate the pharmacodynamic effect, that is, the sedative effect of midazolam, on COMFORT-B scores, we developed an ordinal logistic regression model (Table 3 ). We tested the relation between midazolam concentrations and the probability of the categorized COMFORT-B scores, that is, undersedation (COMFORT-B < 11), adequately sedated (COMFORT-B 11-16), or oversedation (COMFORT-B $>16)$.

Down syndrome was not a significant covariate, since the OF remained 560 after testing this covariate. Including the effect of NRS in the model led to a decrease in OF $(\Delta$ OF $20 ; p<0.001)$ (Supplementary Table 1, Supplemental Digital Content 1, http://links. lww.com/PCC/B533). This implies especially for children who have a NRS for pain greater than or equal 
TABLE 2.

Population Pharmacokinetic Variables of Pharmacokinetic Model for Midazolam in Children and Results of Bootstrap Analysis

\begin{tabular}{|c|c|c|c|}
\hline Variables & $\begin{array}{c}\text { Values Based on } \\
\text { Simple Model (CV in \%) }\end{array}$ & $\begin{array}{l}\text { Values Based on Final } \\
\text { Model (CV in \%) }\end{array}$ & $\begin{array}{l}\text { Mean Bootstrap } \\
\text { Value (CV in \%) }\end{array}$ \\
\hline \multicolumn{4}{|l|}{ Midazolam } \\
\hline $\mathrm{CL}_{1}(\mathrm{~L} / \mathrm{min})$ & $0.020(17.7)$ & $0.019(16.3)$ & $0.019(19.2)$ \\
\hline $\mathrm{CL}_{4}(\mathrm{~L} / \mathrm{min})$ & $0.0015(14.9)$ & $0.0015(20.5)$ & $0.0015(24.9)$ \\
\hline $\mathrm{V}_{1, \text { central }}(\mathrm{L})$ & $1.55(13.6)$ & $1.51(15.6)$ & $1.52(18.7)$ \\
\hline $\mathrm{V}_{2, \text { peripheral }}(\mathrm{L})$ & $12.9(25.9)$ & $16.4(24.5)$ & $18.1(35.7)$ \\
\hline $\mathrm{Q}(\mathrm{L} / \mathrm{min})$ & $0.07(15.5)$ & $0.06(15.4)$ & $0.06(16.1)$ \\
\hline \multicolumn{4}{|l|}{ 1-OH-midazolam } \\
\hline $\mathrm{CL}_{2}(\mathrm{~L} / \mathrm{min})$ & $0.093(17.3)$ & $\begin{array}{c}0.095(14.9)+ \\
0.019(23.5) \times(B W-6.5)\end{array}$ & $\begin{array}{l}0.092(20.5)+ \\
0.017(36.3) \times(B W-6.5)\end{array}$ \\
\hline$M F\left(\right.$ in $\left.V_{3}=V_{1, \text { central }} \times M F\right)$ & 0.9 fixed & 0.9 fixed & 0.9 fixed \\
\hline \multicolumn{4}{|l|}{ 1-OH-midazolam-glucuronide } \\
\hline $\mathrm{V}_{4,1-\mathrm{OHG} \text { central }}=\mathrm{V}_{5-\mathrm{OHG} \text { peripheral }}(\mathrm{L})$ & $0.13(32.9)$ & $0.12(29.7)$ & $0.11(31.8)$ \\
\hline $\mathrm{Q}_{1}(\mathrm{~L} / \mathrm{min})$ & $0.0036(43.7)$ & $0.0032(37.9)$ & $0.0033(39.7)$ \\
\hline $\mathrm{CL}_{3}(\mathrm{~L} / \mathrm{min})$ & $0.0086(18.3)$ & $0.0081(16.9)$ & 0.0079 (19.7) \\
\hline
\end{tabular}

4-OH-midazolam

$$
\begin{aligned}
& \mathrm{V}_{6}=\mathrm{V}_{3}(\mathrm{~L}) \\
& \mathrm{CL}_{5}(\mathrm{~L} / \mathrm{min})
\end{aligned}
$$$$
0.039(18.4)
$$$$
0.038(24.5)
$$$$
0.039(29.5)
$$

Interindividual variability

$\begin{array}{llll}\omega \mathrm{CL}_{1}{ }^{2} & 0.34(37.0) & 0.24(36.0) & 0.24(45.6) \\ \omega \mathrm{V}_{1, \text { central }}{ }^{2} & 1.13(29.3) & 1.15(28.3) & 1.15(39.4) \\ \omega_{2, \text { peripheral }}{ }^{2} & 1.29(34.0) & 1.29(33.7) & 1.39(38.5) \\ \omega \mathrm{CL}_{2}{ }^{2} & 0.29(30.0) & 0.11(29.0) & 0.12(42.9) \\ \omega \mathrm{CL}_{3}{ }^{2} & 0.37(33.9) & 0.30(45.2) & 0.28(52.4) \\ \omega \mathrm{CL}_{1} \mathrm{CL}_{3}{ }^{2} & 0.27(34.9) & 0.19(43.7) & 0.17(60.1)\end{array}$

Residual variability

$\begin{array}{llll}\sigma^{2}{ }_{\text {(midazolam) }} & 0.37(22.8) & 0.37(23.2) & 0.35(23.7) \\ \sigma^{2}{ }_{(1-\mathrm{OH})} & 0.20(24.3) & 0.21(24.3) & 0.20(25.3) \\ \sigma^{2}{ }_{(1-\mathrm{OHG})} & 0.17(17.2) & 0.18(17.8) & 0.18(19.9) \\ \sigma^{2}{ }_{(4-\mathrm{OH})} & 0.17(16.1) & 0.19(16.5) & 0.18(19.0)\end{array}$

OF $(-2 L L)$

0.7

$-8.069$

$\mathrm{BW}=$ bodyweight, $\mathrm{CL} 1=$ clearance of midazolam to 1-OH-midazolam, $\mathrm{CL} 2=$ clearance of 1-OH-midazolam to 1-OH-midazolamglucuronide, $\mathrm{CL} 3=$ clearance of 1-OH-midazolam-glucuronide, $\mathrm{CL} 4=$ clearance of midazolam to 4-OH-midazolam, $\mathrm{CL5}=\mathrm{clearance}$ of $4-\mathrm{OH}$-midazolam, $\mathrm{CV}=$ coefficient of variation, $\mathrm{MF}=$ multiplication factor, $\mathrm{Q}=$ intercompartmental clearance of midazolam, $\mathrm{Q} 1$ = intercompartmental clearance of $1-\mathrm{OH}$-midazolam-glucuronide, $\mathrm{V}=$ volumes of distribution, $\omega 2=$ variance, the square root of the exponential variance of $\eta-1$ is the percentage of interindividual variability in the variables, $\sigma 2=$ proportional intraindividual variance, $\mathrm{OF}(-2 \mathrm{LL})=$ objective function, $1-\mathrm{OH}=1-\mathrm{OH}$ - midazolam, $1-\mathrm{OHG}=1-\mathrm{OH}$-midazolam-glucuronide. 

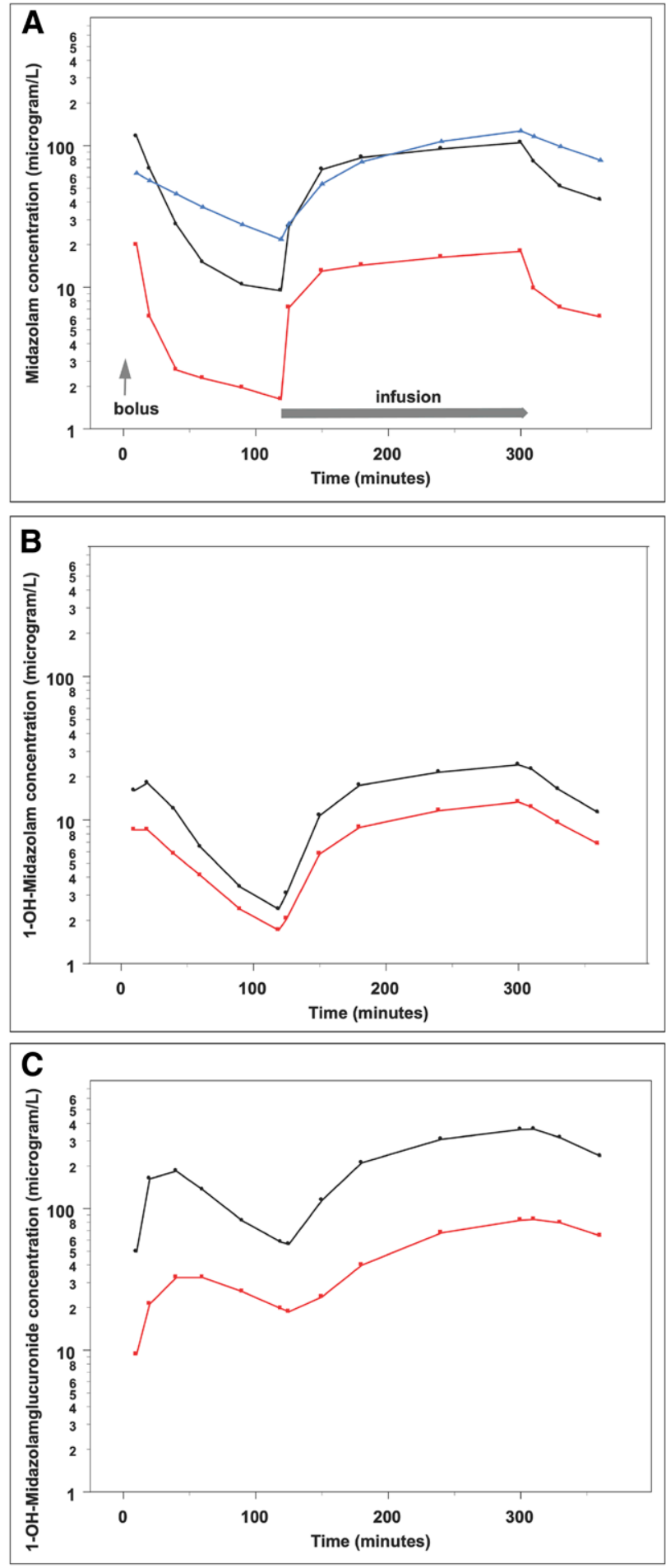

Figure 1. Midazolam $(\mathbf{A})$ and metabolite $(\mathbf{B}, \mathbf{C})$ concentration versus time in children with a bodyweight of $6 \mathrm{~kg}$ after a bolus dose of $0.05 \mathrm{mg} / \mathrm{kg}$ followed by a continuous infusion that was started $2 \mathrm{hr}$ after the bolus dose at a rate of the starting dose $0.06 \mathrm{mg} / \mathrm{kg} / \mathrm{hr}$ during $3 \mathrm{hr}$. The simulations were based on the current study for children after cardiac surgery (circles and black line), for the published study of Vet et al (14), for critically ill children with $\mathrm{C}$-reactive protein $35 \mathrm{mg} / \mathrm{L}$ and one failing organ (triangles and blue line), and for the study of Peeters et al (15) for nonventilated children after craniofacial surgery (squares and red line). Vet et al (14) did not determine metabolite concentrations. to 4 , there is a higher probability of undersedation at lower midazolam concentrations (Fig. 2B). In children with a NRS score less than 4, we did not observe this effect of midazolam concentrations on the probability of undersedation (Fig. 2A).

\section{DISCUSSION}

\section{Sedation With Midazolam in Children With and Without Down Syndrome}

This study shows that the dosing of midazolam does not need to be adjusted for children with Down syndrome. We found that neither the time before additional sedation was required nor the cumulative midazolam dose did statistically significantly differ between children with and without Down syndrome. We developed a population pharmacokinetic model that describes the distribution and clearance of midazolam and its metabolites 1-OH-midazolam, 4-OH-midazolam and 1-OH-midazolam-glucuronide. Down syndrome was not a significant covariate on the pharmacokinetics of midazolam.

Furthermore, the pharmacodynamic analysis showed no difference for the sedative effect of midazolam between children with and without Down syndrome.

\section{Pharmacokinetics of Midazolam After Cardiac Surgery}

In the present study, the concentrations of midazolam and its metabolites compared well with those in the previously published model by Vet et al (14) for children of different ages and different underlying diseases admitted to the ICU.

Vet et al (14) found that disease severity, expressed as number of failing organs, as well as CRP as a marker of inflammation, significantly affect critically ill children's midazolam clearance. In that study, the median CRP was $32 \mathrm{mg} / \mathrm{L}$ (IQR $0.3-385 \mathrm{mg} / \mathrm{L}$ ). The median postoperative CRP value in our study was $34 \mathrm{mg} / \mathrm{L}$, but CRP was not a significant covariate in the population pharmacokinetic analysis. Nguyen et al (16) reported that elevated levels of proinflammatory cytokines associated with infection and inflammation can modulate cytochrome P450 enzymes; interleukin-6 exposure up-regulated acute phase proteins (CRP, alpha-1-acid glycoprotein) and down-regulated CYP3A4. The proposed mechanism mediating cytochrome suppression 
TABLE 3.

Population Pharmacodynamic Variables for the Basic and Final Model With Numeric Rating Scale Less Than 4 and Numeric Rating Scale Greater Than or Equal to 4 as Covariate Based on the COMFORTBehavioral Score Categorized

\begin{tabular}{|c|c|c|}
\hline Variables & $\begin{array}{c}\text { Basic } \\
\text { COMFORT-B } \\
\text { Categorized, } \\
\text { Mean (CV\%) }\end{array}$ & $\begin{array}{c}\text { Final } \\
\text { COMFORT-B } \\
\text { Categorized, } \\
\text { Mean (CV\%) }\end{array}$ \\
\hline \multicolumn{3}{|l|}{ Fixed effects } \\
\hline$\theta_{1}$ & $1.2(22.5)$ & $1.5(17.4)$ \\
\hline$\theta_{2}$ & $-4.0(-8.0)$ & $-4.2(-7.9)$ \\
\hline $\begin{array}{l}\theta_{3} \text { (midazolam); } \\
\theta 4 \text { (when Numeric } \\
\text { Rating Scale } \geq 4 \text { ) }\end{array}$ & 0.0020 (52.9) & $\begin{array}{c}0.0019(58.2) \\
-1.8(-30.6)\end{array}$ \\
\hline \multicolumn{3}{|l|}{ Interindividual variability } \\
\hline$\omega^{2}$ & $1.22(39.8)$ & $1.45(40.9)$ \\
\hline \multicolumn{3}{|l|}{ Performance measures } \\
\hline$-2 L L$ & 560.69 & 540.411 \\
\hline
\end{tabular}

COMFORT-B $=$ COMFORT-Behavioral, $\mathrm{CV}=$ coefficient of variation, $-2 \mathrm{LL}=$ objective function, $\theta 1, \theta 2=$ cut-off points, $\theta 3=$ magnitude of the midazolam effect, $\theta 4=$ magnitude of the Numeric Rating Scale (NRS) effect (with $0=N R S<4$ and $1=N R S \geq 4$ ), $\omega 2=$ variance of the interindividual variability.

is most likely a result of direct interaction with cell surface receptors. As cardiopulmonary bypass is a potent trigger for a systemic inflammatory response, in our study, such an effect did not come unsuspectedly (17). Possible explanations for a lack of influence of CRP as a covariate in our study are the small range in CRP values, delayed modulation of the cytochrome $\mathrm{P} 450$ enzymes, and the fact that midazolam was started directly after the hit (cardiopulmonary bypass), during a relatively short study period $(<72 \mathrm{hr})$. The estimated degradation half-life of CYP3A4 is between 26 and 144 hours (18).

The majority of the 4-OH-midazolam concentrations were below the LLQ $(2 \mathrm{ng} / \mathrm{mL})$. The reported metabolic conversion fraction of midazolam to 4-OH-midazolam is 0.03 (19). It is understandable; therefore, that in view of the relatively low midazolam doses in this study, most of the 4-OH-midazolam concentrations were below the LLQ.
We identified bodyweight as a significant covariate on the glucuronidation clearance of 1-OH-midazolam. The glucuronidation of 1-OH-midazolam is mediated by UGT2B4, 2B7, and 1A4. Activity of these enzymes reaches adult levels within the first 1-2 years after birth. This would imply that bodyweight may be a surrogate descriptor for the ontogeny of drug glucuronidation in this population (20).

\section{Pharmacodynamics of Midazolam After Cardiac Surgery}

We found that the presence of Down syndrome had no influence on the pharmacodynamics of midazolam. In addition, a therapeutic effect of midazolam on COMFORT-B scores was hard to detect. We have compared all the scores before and after starting midazolam, looked at the individual plots displaying the COMFORT-B scores and midazolam levels over time (Supplementary Fig. 5, Supplemental Digital Content 1, http://links.lww.com/PCC/B533), and the probability for a particular COMFORT-B score to be a function of the midazolam concentration (Fig. 2). These concerted efforts revealed only a marginal effect of midazolam concentrations on the COMFORT-B scores. The pharmacodynamic model showed a lower probability for undersedation at higher midazolam concentrations. The probability of undersedation was higher when the NRS score was greater than or equal to 4 (Fig. 2B).

Why did we find only a marginal therapeutic effect of midazolam on COMFORT-B scores in this study? First, this may be due to the relatively low dosing of midazolam. Given that all subjects were receiving a morphine infusion as well, and in most cases the midazolam was administered as a bolus, a steady, therapeutic midazolam level may not have been indicated. Since undersedation was more common if NRS was greater than or equal to 4 (Fig. $2 B$ ) compared with NRS score of less than 4 (Fig. 2A), this confirms the overlap between the emotional states of pain and distress in these postoperative children. Second, little is known on the duration of the effect of midazolam on the pharmacodynamic outcome variable: the COMFORT-B scores. It may well be that, in the interval between administration of additional sedation and scoring, the stimulus for the additional sedative requirement (i.e., mechanical ventilation) had been removed. In theory, continuous monitoring of pain and distress levels would be preferable over nurses' assessments every 4-6 


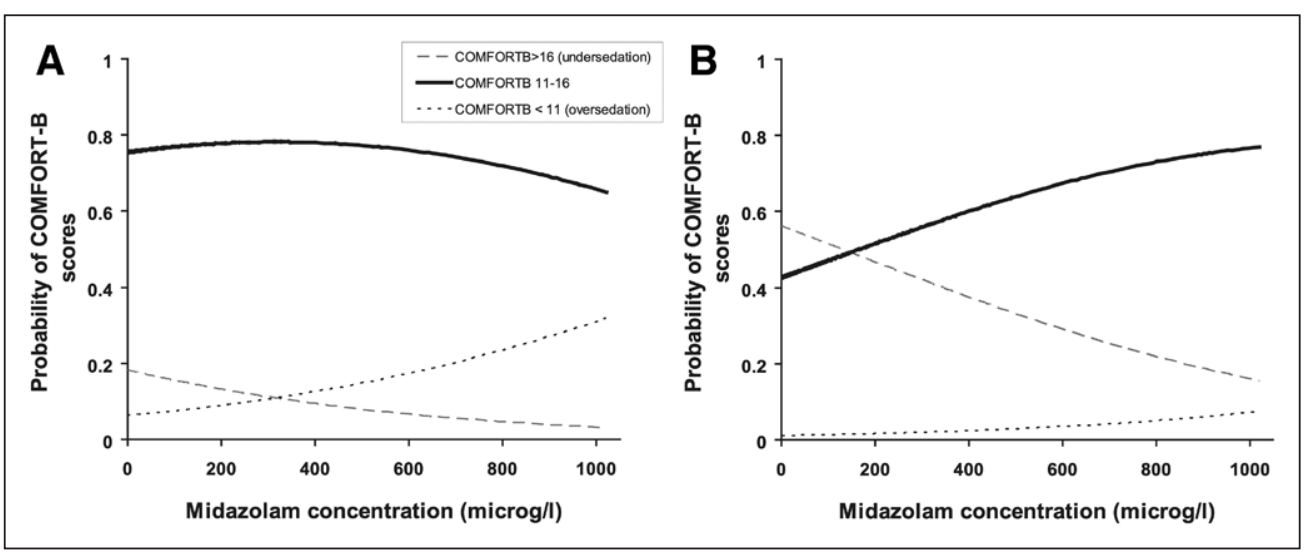

Figure 2. Probability of COMFORT-behavioral (COMFORT-B) score categorized as undersedation (COMFORT-B < 11), adequately sedated (COMFORT-B 11-16), or oversedation COMFORT B $>16)$, as a function of midazolam concentration ( $\mu \mathrm{g} / \mathrm{L})$. A, Shows the results when the Numeric Rating Score (NRS) for pain is less than 4. B, Shows the results when the NRS for pain is greater than or equal to 4 . and had a longer length of stay.

After completion of our study, a "new" (and still off-label) player appeared in the field of analgosedation after pediatric cardiac surgery: dexmedetomidine. This sedative might be less suitable for children with Down syndrome, however, given the greater number of adverse cardiac events reported with dexmedetomidine in this population (25).

Future studies should hours. Unfortunately, a method to continuously monitor pain and distress levels remains to be identified (21). An alternative to modelling COMFORT-B scores is the analysis of time to remedication events. For example, Elkomy et al (22) showed by analyzing the time to morphine remedication events after cardiac surgery in children that the sensitivity to morphine analgesia decreases with age and that higher doses are not incrementally effective.

\section{Optimal Analgosedation After Cardiac Surgery}

In this study, the children received morphine and midazolam according to an algorithm based on regular pain and distress assessments by the nursing staff. Donnelan et al (23) showed recently that the implementation of an algorithm reduced opioid and benzodiazepine dosing in children after cardiac surgery.

More than two thirds of the subjects in our study received midazolam at some point during their intensive care admission, and only half of them needed a midazolam infusion. This finding supports the practice of first addressing undersedation with a midazolam bolus, rather than proceeding immediately with continuous midazolam infusion. This is also supported by the findings of a randomized controlled trial by Penk et al (24) in children after cardiac surgery. It was found that pain was not better controlled with the addition of continuous infusions of morphine and midazolam when compared with intermittent dosing only. Still, the group with continuous infusions had received a significantly higher total dosage of these medications be designed to answer the question of what is the optimal regimen of both maintenance and rescue doses of midazolam to establish an adequate level of comfort during intensive care admission. Data reflecting hemodynamic side-effects as well as the impact on sleep and delirium of such regimens must be captured (8).

\section{Limitations}

The children who required midazolam had a longer stay in the ICU and had been mechanically ventilated for a longer time. The observational nature of the study makes it difficult to draw conclusions on the requirements for midazolam after cardiac surgery.

Furthermore, since only children for elective cardiac surgery were included in this study, overall the duration of intensive care admission was relatively short, that is, a median of 4 days for the midazolam group and a median of 2 days for the children who did not require midazolam. This resulted in relatively low midazolam dosages and consequently low midazolam levels. Delirium assessments were not part of the study protocol, and therefore, we cannot report the incidence of postoperative delirium in the children with and without Down syndrome.

\section{CONCLUSIONS}

The pharmacokinetic and pharmacodynamic results from this study provide no evidence to support 
altered dosing of midazolam in children with Down syndrome after cardiac surgery. This study confirms the observation that the majority of children with and without Down syndrome require sedation on top of morphine analgesia during intensive care admission after cardiac surgery. However, in both groups, the pharmacodynamic analysis revealed only a marginal effect of midazolam concentrations on the COMFORT-B scores.

\section{ACKNOWLEDGMENTS}

We would like to thank Claire Magner, $\mathrm{PhD}$, Department of Anesthesia and Critical Care Medicine, Crumlin, Dublin 12, Ireland, for her support with the data collection.

1 Department of Intensive Care, Erasmus University Medical Center, Rotterdam, the Netherlands.

2 Intensive Care and Department of Pediatric Surgery, Erasmus University Medical Center - Sophia Children's Hospital, Rotterdam, the Netherlands.

3 Leiden Academic Centre for Drug Research, Division of Systems Biomedicine and Pharmacology, Leiden University, Leiden, the Netherlands

4 Department of Anaesthesia and Critical Care Medicine, Our Lady's Children's Hospital, Dublin, Ireland

5 Department of Pharmacy, Academic UMC, Amsterdam, the Netherlands.

6 Department of Internal Medicine - Section of Nursing Science, Erasmus University Medical Center, Rotterdam, the Netherlands.

7 Department of Clinical Pharmacy, St. Antonius Hospital, Nieuwegein, the Netherlands.

Supplemental digital content is available for this article. Direct URL citations appear in the printed text and are provided in the $H T M L$ and PDF versions of this article on the journal's website (http://journals.lww.com/pccmjournal).

Supported, in part, by a grant (F/12/4) from the National Children's Research Centre, Dublin, Ireland.

Drs. Valkenburg's and Peeters's institutions received funding from National Children's Research Centre, Dublin, Ireland. The remaining authors have disclosed that they do not have any potential conflicts of interest.

For information regarding this article, E-mail: a.valkenburg@erasmusmc.nl

This work was performed at Department of Anesthesia and Critical Care Medicine, Our Lady's Children's Hospital, Crumlin, Dublin 12, Ireland.

\section{REFERENCES}

1. Down JL: On Some of the Mental Affections of Childhood and Youth: Being the Lettsomian Lectures Delivered Before the Medical Society of London in 1887. London, United Kingdom, J. \& A. Churchill, 1887

2. Terada Y, Tachibana K, Takeuchi M, et al: [Comparison of sedative and analgesic requirements in children with and without down syndrome following pediatric cardiac surgery]. Masui 2016; 65:56-61

3. Gakhal B, Scott CS, MacNab AJ: Comparison of morphine requirements for sedation in Down's syndrome and nonDown's patients following paediatric cardiac surgery. Paediatr Anaesth 1998; 8:229-233

4. Valkenburg AJ, Calvier EA, van Dijk M, et al: Pharmacodynamics and pharmacokinetics of morphine after cardiac surgery in children with and without down syndrome. Pediatr Crit Care Med 2016; 17:930-938

5. Goot BH, Kaufman J, Pan Z, et al: Morphine pharmacokinetics in children with down syndrome following cardiac surgery. Pediatr Crit Care Med 2018; 19:459-467

6. Ni She R, Filan PM: Trisomy 21-incidence and outcomes in the first year, in Ireland today. Ir Med J 2014; 107:248-249

7. Bergstrom S, Carr H, Petersson G, et al: Trends in congenital heart defects in infants with down syndrome. Pediatrics 2016;138:e20160123

8. Walker T, Kudchadkar SR: Pain and sedation management: 2018 update for the Rogers' textbook of pediatric intensive care. Pediatr Crit Care Med 2019; 20:54-61

9. Valkenburg AJ, Boerlage AA, Ista $E$, et al: The COMFORTbehavior scale is useful to assess pain and distress in 0- to 3-yearold children with Down syndrome. Pain 2011; 152:2059-2064

10. Ista E, van Dijk M, Tibboel D, et al: Assessment of sedation levels in pediatric intensive care patients can be improved by using the COMFORT "behavior" scale. Pediatr Crit Care Med 2005; 6:58-63

11. Bai J, Hsu L, Tang Y, et al: Validation of the COMFORT behavior scale and the FLACC scale for pain assessment in Chinese children after cardiac surgery. Pain Manag Nurs 2012; 13:18-26

12. Carnevale FA, Razack S: An item analysis of the COMFORT scale in a pediatric intensive care unit. Pediatr Crit Care Med 2002; 3:177-180

13. van Dijk M, de Boer JB, Koot HM, et al: The reliability and validity of the COMFORT scale as a postoperative pain instrument in 0 to 3-year-old infants. Pain 2000; 84:367-377

14. Vet NJ, Brussee JM, de Hoog M, et al; SKIC (Dutch collaborative PICU research network): Inflammation and organ failure severely affect midazolam clearance in critically ill children. Am J Respir Crit Care Med 2016; 194:58-66

15. Peeters MY, Prins SA, Knibbe CA, et al: Pharmacokinetics and pharmacodynamics of midazolam and metabolites in nonventilated infants after craniofacial surgery. Anesthesiology 2006; 105:1135-1146 
16. Nguyen TV, Ukairo O, Khetani SR, et al: Establishment of a hepatocyte-kupffer cell coculture model for assessment of proinflammatory cytokine effects on metabolizing enzymes and drug transporters. Drug Metab Dispos 2015; 43:774-785

17. Hirleman E, Larson DF: Cardiopulmonary bypass and edema: Physiology and pathophysiology. Perfusion 2008; 23:311-322

18. Ince I, de Wildt SN, Peeters MY, et al: Critical illness is a major determinant of midazolam clearance in children aged 1 month to 17 years. Ther Drug Monit 2012; 34:381-389

19. Zuppa AF, Conrado DJ, Zane NR, et al: Midazolam dose optimization in critically ill pediatric patients with acute respiratory failure: A population pharmacokinetic-pharmacogenomic study. Crit Care Med 2019; 47:e301-e309

20. Krekels $E H$, Danhof M, Tibboel D, et al: Ontogeny of hepatic glucuronidation; methods and results. Curr Drug Metab 2012; 13:728-743

21. Harris J, Ramelet AS, van Dijk M, et al: Clinical recommendations for pain, sedation, withdrawal and delirium assessment in critically ill infants and children: An ESPNIC position statement for healthcare professionals. Intensive Care Med 2016; 42:972-986

22. Elkomy MH, Drover DR, Galinkin JL, et al: Pharmacodynamic analysis of morphine time-to-remedication events in infants and young children after congenital heart surgery. Clin Pharmacokinet 2016; 55:1217-1226

23. Donnellan A, Sawyer J, Peach A, et al: Reducing exposure to opioid and benzodiazepine medications for pediatric cardiac intensive care patients: A quality improvement project. Pediatr Crit Care Med 2019; 20:340-349

24. Penk JS, Lefaiver CA, Brady CM, et al: Intermittent versus continuous and intermittent medications for pain and sedation after pediatric cardiothoracic surgery; A randomized controlled trial. Crit Care Med 2018; 46:123-129

25. Ueno K, Ninomiya $Y$, Shiokawa $N$, et al: Dexmedetomidine is associated with an increased incidence of bradycardia in patients with trisomy 21 after surgery for congenital heart disease. Pediatr Cardiol 2016; 37:1228-1234 